\title{
Evaluación de la influencia e impacto del análisis de video en el nivel de comprensión de gráficos en cinemática: una alternativa de bajo costo y de fácil implementación
}

\author{
CÉSAR Rosas ECHEVARría ${ }^{1}$ \\ Jorge Hilario CÁRDENAS ${ }^{2}$ \\ Roberto Perales Flores ${ }^{3}$ \\ Alberto Cerna Cueva ${ }^{4}$ \\ Justina Isabel Prado Juscamaita ${ }^{5}$
}

ReCibido: 09/09/2019 AcEPtAdo: 21/01/2020 Publicado: 16/10/2020

\begin{abstract}
RESUMEN
Este artículo es un estudio que evidencia los beneficios de implementar el análisis de video (AV) en clase de Física. El objetivo de la investigación es demostrar que la aplicación del $\mathrm{AV}$, además de ser una técnica de bajo costo y de fácil uso, mejora significativamente el nivel de comprensión de gráficos en cinemática (NCGC). En el trabajo se comparan los resultados de la parte práctica de tema de cinemática en una clase tradicional y en una con AV, después de aplicar el TUG-K (Test of Understanding Graphs in Kinematics), para evaluar la influencia del AV en el NCGC a 60 alumnos seleccionados de la Facultad de Ingeniería de la Universidad de Huánuco (30 para cada grupo). Los resultados muestran que existe una fuerte correlación biserial puntual $\left(r_{b p}=0.809\right)$ altamente significativa $(p<0.0001)$ entre estas dos variables, que el AV tiene un impacto medio en el NCGC (Hake = $32.5 \%$ ) y que el NCGC es significativamente mayor ( $p$ $<0.0001$ ) cuando se aplica AV en comparación con la clase tradicional.
\end{abstract}

Palabras claves: análisis de video; nivel decomprensión de gráficos en cinemática; TUG-K; correlación biserial puntual; seguimiento de punto.

\section{INTRODUCCIÓN}

La capacidad para trabajar con gráficos es una habilidad básica del científico (Beichner, 1994); sin embargo, existen estudios que demuestran que esta no es una habilidad de la mayoría de los alumnos que llevan cinemática en su formación académica. Según Cid (2011), existe una correlación entre el rendimiento en los campos de ciencia, tecnología, ingeniería y matemáticas, y la capacidad de trabajar con gráficos. Por otra parte, según Pontes (2005), es sabido que existe una creciente influencia de las tecnologías de la información y comunicación (TIC) en la educación científica a nivel secundario y universitario.

Una de las maneras de evaluar el nivel de comprensión de gráficos en cinemática (NCGC) es por medio la prueba de comprensión de gráficos en cinemática o Test of Understanding Graphs in Kinematics (conocido por su sigla TUG-K), propuesta por Beichner (1994), quien la aplicó a 524 estudiantes de secundaria y universitarios, y obtuvo una media de 8.5 de 21 preguntas solo un $40 \%$ de aciertos. Del mismo modo, Ribotta, Pesetti y Pereyra (2009) han evidenciaado el bajo NCGC con el TUG-K, puesta en práctica en 62 alumnos que cursaban Física I, en la Facultad de Ingeniería y Ciencias Económico Sociales de la Universidad Nacional de San Luis (Argentina); en esta prueba el resultado de aciertos fue de $36.83 \%$.

Ingeniero industrial por la Universidad Nacional Hermilio Valdizán (Huánuco, Perú). Magíster en Gestión de Proyectos. Actualmente, es docente contratado de dicha institución y de la Universidad de Huánuco. (Huánuco, Perú).

ORCID: https://orcid.org/0000-0002-3256-3102

E-mail: crosas@unheval.edu.pe

2 Doctor en Gestión Empresarial por la Universidad Nacional Hermilio Valdizán (Huánuco, Perú). Actualmente, es docente principal de dicha institución. (Huánuco, Perú).

ORCID: https://orcid.org/0000-0001-6627-6489

E-mail: jhilario@unheval.edu.pe

3 Magíster en Educación y Planeamiento de la Universidad Nacional Hermilio Valdizán (Huánuco, Perú). Actualmente, es profesor principal de dicha institución. (Huánuco, Perú). ORCID: https://orcid.org/0000-0002-2502-9593

E-mail: rperales@unheval.edu.pe

4 Ingeniero ambiental en la Universidad Nacional Agraria de la Selva (Tingo María, Perú). Es consultor independiente. (Tingo María, Perú)

ORCID: https://orcid.org/0000-0001-7448-558X

E-mail: franco.cerna@unas.edu.pe

5 Doctora en Ciencias de la Salud por la Universidad Nacional Hermilio Valdizán (Huánuco, Perú). Actualmente, es docente principal de dicha institución. (Huánuco, Perú).

ORCID:https://orcid.org/0000-0002-6558-4233

E-mail: jprado@unheval.edu.pe 
Por otro lado, el método de enseñanza tradicional prescinde de los recursos tecnológicos, lo que ocasiona que sean limitadas las representaciones externas de los conceptos teóricos, debido a las restricciones de las capacidades sensoriales humanas. Es por ello que existen estudios que han demostrado que los procesos de aprendizaje basado en experimentos (ABE) mejoran la comprensión de gráficos cinemáticos; un ejemplo de esto es el trabajo de Sandoval, Mora y Suárez (2017). Dichos investigadores aplicaron el TUG-K a cuatro grupos de estudiantes (tres con el método de enseñanza tradicional y un grupo con $A B E$ ), los cuales eran de las especialidades de Ingeniería Ambiental, Industrial o Petrolera y estudiaban en el Instituto Superior Tecnológico de Comalcalco o en la Universidad Politécnica del Golfo de México; el resultado fue una ganancia de Hake superior al $40 \%$. Este experimento demostró que el $\mathrm{ABE}$ mejora significativamente el NCGC.

En cinemática, la forma directa de interactuar y experimentar con el problema es mediante la adquisición de datos en tiempo real de la posición-tiempo de un objeto, para su posterior análisis, mediante modelos físicos; sin embargo, esta adquisición de datos solo es posible si se utilizan herramientas tecnológicas. Una de estas herramientas es el análisis de video (AV), el cual es una serie de imágenes fotográficas estáticas que, cuando se muestran en orden secuencial, proporcionan una imagen de movimiento, lo que permite la construcción de la posición-tiempo (Navarrete, Almaguer, Navarrete y Flores, 2015). Los fundamentos del AV no son conceptos nuevos, pero su aplicación a la enseñanza se ha desarrollado recientemente. Hace algunos años, la aplicación de los recursos tecnológicos en los procesos de enseñanza y aprendizaje eran vistos como poco convencionales para su generalización, debido a su complejidad y precio; ahora, es viable el empleo de herramientas de fácil acceso y bajo costo.

Finalmente, dentro de las investigaciones acerca del proceso de enseñanza utilizando la técnica de AV para la comprensión de gráficos en cinemática, se encuentra la de Navarrete et al. (2015), quienes trabajaron con seis grupos de estudiantes del curso de Física del laboratorio de mecánica de las carreras de Ingeniería Industrial y de Ingeniería Química del Centro Universitario de Ciencias Exactas e Ingenierías de la Universidad de Guadalajara. En dicha investigación, se aplicó el TUG-K antes y después de la integración del AV en las clases durante el semestre febrero-junio de 2013 , y se obtuvo como resultado una ganancia de Hake promedio de
$42.86 \%$, por lo que se concluyó que la integración del AV en las clases de Física mejoran el NCGC. Otro trabajo en el que se aplica el análisis de video es el ya citado estudio de Ribotta et al, (2009), quienes trabajaron con 62 estudiantes del curso Física I de la FICES de la UNSL a los que se les realizó el TUG-K, al inicio y final de la instrucción, divididos en 3 grupos experimentales (21 alumnos de control, 20 aplicando DataStudio y 21 aplicando VideoPoint), obteniendo como resultados una ganancia de Hake del $5,0 \%, 21,8 \%$ y $17,8 \%$ respectivamente. De ese modo, se concluyó que la aplicación de las TIC contribuye a mejorar los aspectos pedagógicos-didácticos en la formación de ingenieros.

\section{MATERIALES Y MÉTODOS}

\section{Delimitación de la población y muestra}

Para evaluar el impacto del TUG-K en la interpretación de gráficos en cinemática, esta investigación comparó una clase tradicional con una clase que integra el AV. Se seleccionaron 30 alumnos de Arquitectura y 30 de Ingeniería Civil, para la aplicación del TUG-K en una clase tradicional y en una en la que se integró el AV, respectivamente; ambos grupos estaban compuestos por estudiantes del primer ciclo que cursaban Física I en la Facultad de Ingeniería de la Universidad de Huánuco. El número de alumnos por grupo resultó de un proceso de depuración, ya que la cantidad de estudiantes inicialmente fue mayor; pero solo se seleccionaron a los alumnos que asistían regularmente a las clases previas al tema de cinemática y a los que más participaron durante la clase de ese tema.

\section{Implementación de la técnica del AV}

Siguiendo el estudio de Meneses, Niño y García (2017), el procedimiento e implementación de la técnica del $\mathrm{AV}$ constó de lo siguiente:

- Fondo de contraste: la técnica del AV trabaja con los píxeles de cada fotograma, por esta razón, para facilitar el contraste del objeto de estudio (en este caso una pelota de hule) y el fondo, se instaló una tela negra rectangular de $2 \times 3 \mathrm{~m}$ en un marco de madera.

- Establecimiento de eje de coordenadas: esto se realizó identificando la esquina inferior izquierda del fondo de la tela negra. Aunque los lanzamientos se realizaron en el primer cuadrante del eje, esto sirvió como referencia del movimiento.

- Barra de calibración: para esto se utilizó una barra de madera de color blanco de 1 metro de longitud, 
la cual se ubicó en uno de los costados de la tela negra. De esta forma, independientemente de la distancia a la que se grabó el video, se pudo calcular los desplazamientos en el plano xy

- Detectar la presencia de un objeto de color (en este caso la pelota de hule) usando técnicas de visión por computadora: para ello, fue de ayuda colocar el fondo negro para que el contraste facilite el proceso discriminativo de selección de color con la librería Tracking de OpenCV. Se utilizó el lenguaje de programación Phyton 2.7.

- Rastrear la pelota a medida que se movía en los cuadros de video: para ello, se utilizó una grabación de una cámara de celular. La información de la posición de la pelota de hule es puntual, pero esta es un objeto que posee superficie; entonces, para superar ello, se trabajó con el centro de la figura de la pelota, la cual fue calculada a partir de sus píxeles. El seguimiento del video se hizo para intervalos de cada 100 milisegundos, y esta información fue exportada a Excel, donde se realizaron las gráficas (s vs. t), (v vs. t) y (a vs. t).

\section{Preparación de clase de estudio}

A continuación, la Figura 1 muestra un esquema de las clases de estudio impartidas.

\section{TUG-K}

El TUG-K tiene como fin ser una herramienta de diagnóstico del NCGC. Esta prueba posee siete objetivos de evaluación y tres preguntas por objetivo, lo que hace un total de 21 preguntas. En la Tabla 1 se pueden ver los detalles:

\section{Diseño estadístico}

Con la finalidad de determinar si existe relación entre la clase recibida (tradicional o con AV) y el porcentaje de aciertos del TUG-K, se aplicó el coeficiente de correlación biserial puntual. En general, la correlación biserial es una medida de asociación entre dos variables continuas, las cuales se distribuyen de la misma forma; para facilitar el proceso, una de ellas se dicotomiza (sí-no, falso-verdadero, masculino-femenino, casado-soltero, bueno-malo, entre otros). La variable dicotomizada se supone

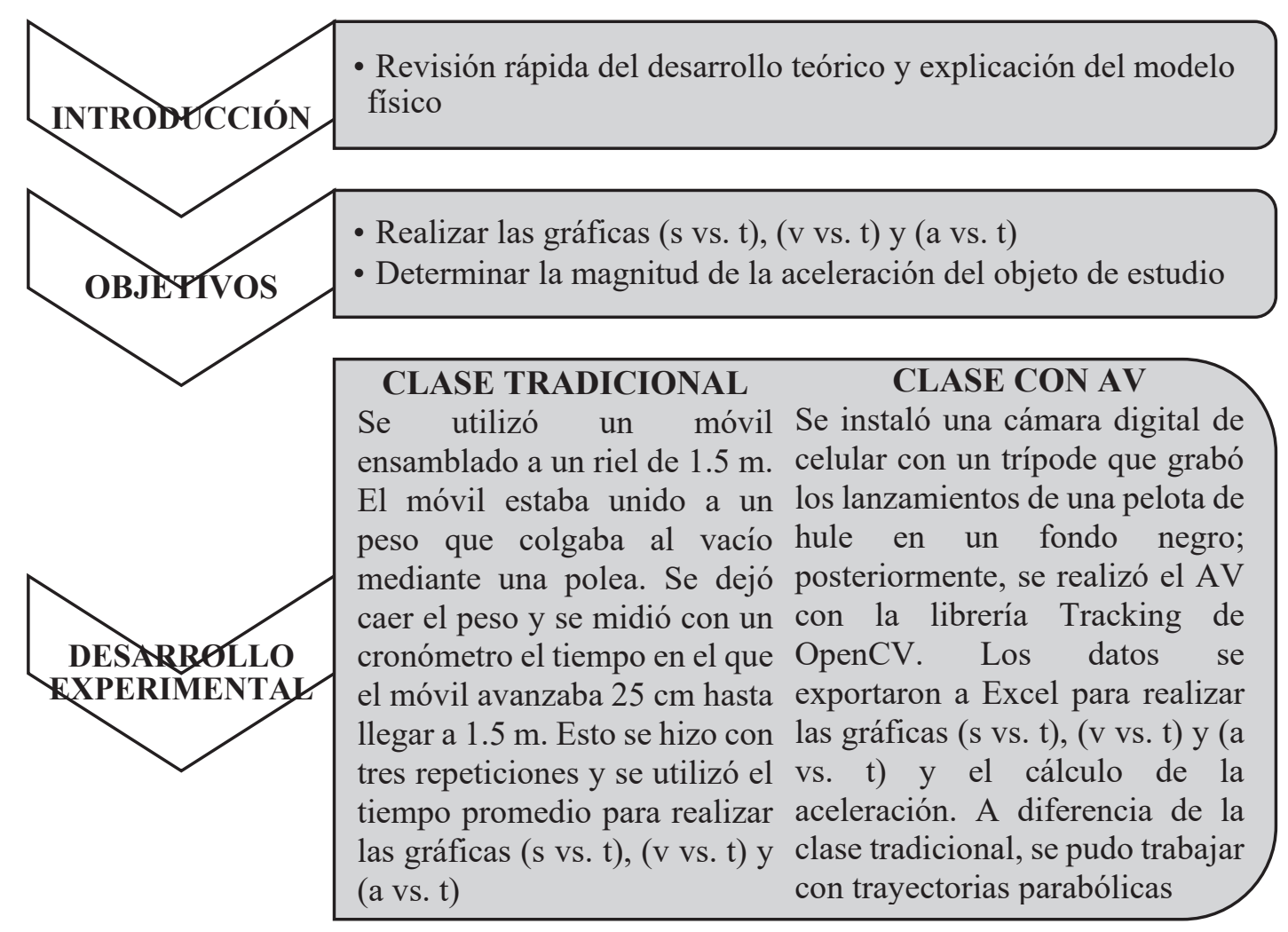

Figura 1. Procedimiento de las clases prácticas de cinemática: la tradicional y la que incluye análisis de video.

Fuente: Elaboración propia. 
Tabla 1. Objetivos del TUG-K.

\begin{tabular}{|c|c|c|}
\hline N. & Dado lo siguiente & El estudiante debe \\
\hline 1 & Gráfico de posición-tiempo & Determinar la velocidad \\
\hline 2 & Gráfico de velocidad-tiempo & Determinar la aceleración \\
\hline 3 & Gráfico de velocidad-tiempo & Determinar el desplazamiento \\
\hline 4 & Gráfico de aceleración-tiempo & Determinar el cambio en la velocidad \\
\hline 5 & Un gráfico de cinemática & Seleccionar otro gráfico correspondiente \\
\hline 6 & Un gráfico de cinemática & Seleccionar la descripción textual \\
\hline 7 & Descripción del movimiento textual & Seleccionar el gráfico correspondiente \\
\hline
\end{tabular}

Fuente: Beichner (1994).

discreta o discontinua cuando se trata de relacionar con la que permanece continua; el coeficiente más apropiado para este propósito es el de correlación biserial puntual $\left(r_{\mathrm{bp}}\right)$ (Elorza, 1999). Para este estudio, la variable dicotomizada será la clase recibida (0 para la clase tradicional y 1 para la clase con AV). El coeficiente de correlación biserial puntual se calculó de la siguiente manera:

$$
r_{b p}=\frac{\bar{X}_{p}-\bar{X}}{S_{x}} \sqrt{\frac{p}{q}}
$$

Donde:

$\overline{\mathrm{X}}_{\mathrm{p}}$ es el promedio de aciertos de los alumnos que recibieron la clase con $\mathrm{AV}$.

$\overline{\mathrm{X}}$ es el promedio total de aciertos de todos los alumnos en ambos grupos.

$\mathrm{S}_{\mathrm{x}}$ es la varianza de la muestra de estudio.

p y q son las proporciones de alumnos que recibieron la clase con AV y la clase tradicional, respectivamente. Como en ambos casos hay 30 alumnos, entonces $p=q=0.5$.

Una vez determinada la correlación, se realizó una prueba de $t$ de Student de una cola a un nivel de significancia de $\alpha=0.05$, para establecer cuál de las clases impartidas (tradicional y con AV) resulta en mayor porcentaje de preguntas acertadas. Por ello, se siguió el mismo procedimiento a nivel de objetivos del TUG-K.

\section{RESULTADOS}

1. Correlación entre la clase impartida y el porcentaje de aciertos de preguntas del TUG-K

Después de que los dos grupos de estudio (tradicional y con AV) recibieron su clase, se les aplicó el TUG-K en un periodo de 45 minutos. En la Figura 2 se muestra la cantidad de aciertos para cada pregunta planteada en el mencionado test.

Por otra parte, en la Tabla 2 se puede observar el porcentaje de aciertos de cada ítem del TUG-K en ambos grupos de estudio. La clase tradicional tuvo en promedio de $21.1 \%$, mientras que la clase con AV alcanzó un promedio de $49.4 \%$. Entre ambos grupos se obtuvo una ganancia de Hake de $32.5 \%$.

Tabla 2. Porcentaje de aciertos por ítem del TUG-K del grupo con la clase tradicional frente al grupo con AV $y$ la ganancia de Hake normalizada.

\begin{tabular}{|c|c|c|c|}
\hline Ítem & Tradicional & Con AV & Ganancia de Hake \\
\hline 1 & $6.7 \%$ & $43.3 \%$ & $39.3 \%$ \\
\hline 2 & $73.3 \%$ & $63.3 \%$ & $-37.5 \%$ \\
\hline 3 & $3.3 \%$ & $23.3 \%$ & $20.7 \%$ \\
\hline 4 & $16.7 \%$ & $46.7 \%$ & $36.0 \%$ \\
\hline 5 & $10.0 \%$ & $43.3 \%$ & $37.0 \%$ \\
\hline 6 & $16.7 \%$ & $60.0 \%$ & $52.0 \%$ \\
\hline 7 & $10.0 \%$ & $73.3 \%$ & $70.4 \%$ \\
\hline 8 & $10.0 \%$ & $20.0 \%$ & $11.1 \%$ \\
\hline 9 & $3.3 \%$ & $33.3 \%$ & $31.0 \%$ \\
\hline 10 & $16.7 \%$ & $80.0 \%$ & $76.0 \%$ \\
\hline 11 & $30.0 \%$ & $53.3 \%$ & $33.3 \%$ \\
\hline 12 & $43.3 \%$ & $36.7 \%$ & $-11.8 \%$ \\
\hline 13 & $33.3 \%$ & $46.7 \%$ & $20.0 \%$ \\
\hline 14 & $36.7 \%$ & $83.3 \%$ & $73.7 \%$ \\
\hline 15 & $23.3 \%$ & $56.7 \%$ & $43.5 \%$ \\
\hline 16 & $10.0 \%$ & $83.3 \%$ & $81.5 \%$ \\
\hline 17 & $16.7 \%$ & $50.0 \%$ & $40.0 \%$ \\
\hline 18 & $50.0 \%$ & $53.3 \%$ & $6.7 \%$ \\
\hline 19 & $16.7 \%$ & $26.7 \%$ & $12.0 \%$ \\
\hline 20 & $13.3 \%$ & $43.3 \%$ & $34.6 \%$ \\
\hline 21 & $3.3 \%$ & $16.7 \%$ & $13.8 \%$ \\
\hline
\end{tabular}

Fuente: Elaboración propia.

Según la Tabla 3, el coeficiente de correlación biserial puntual es de 0.809 , un valor cercano a la 
unidad, por lo que se puede afirmar que hay una fuerte correlación positiva y altamente significativa entre el tipo de clase brindada a los alumnos y el porcentaje de aciertos del TUG-K.

\section{Comparación de los porcentajes de aciertos del TUG-K de ambos grupos de estudio}

A continuación, en la Tabla 4, se presenta el porcentaje de aciertos de cada objetivo del TUG-K.

\section{DISCUSIÓN}

\section{Diagnóstico}

El porcentaje de preguntas contestadas correctamente del TUG-K para el grupo que no recibió la clase con AV fue de $21.1 \%$, valor inferior a los resultados de los estudios de Beichner (1994) y Ribotta et al. (2009), los cuales obtuvieron porcentajes de aciertos de $40 \%$ y $36.83 \%$, respectivamente. Esto

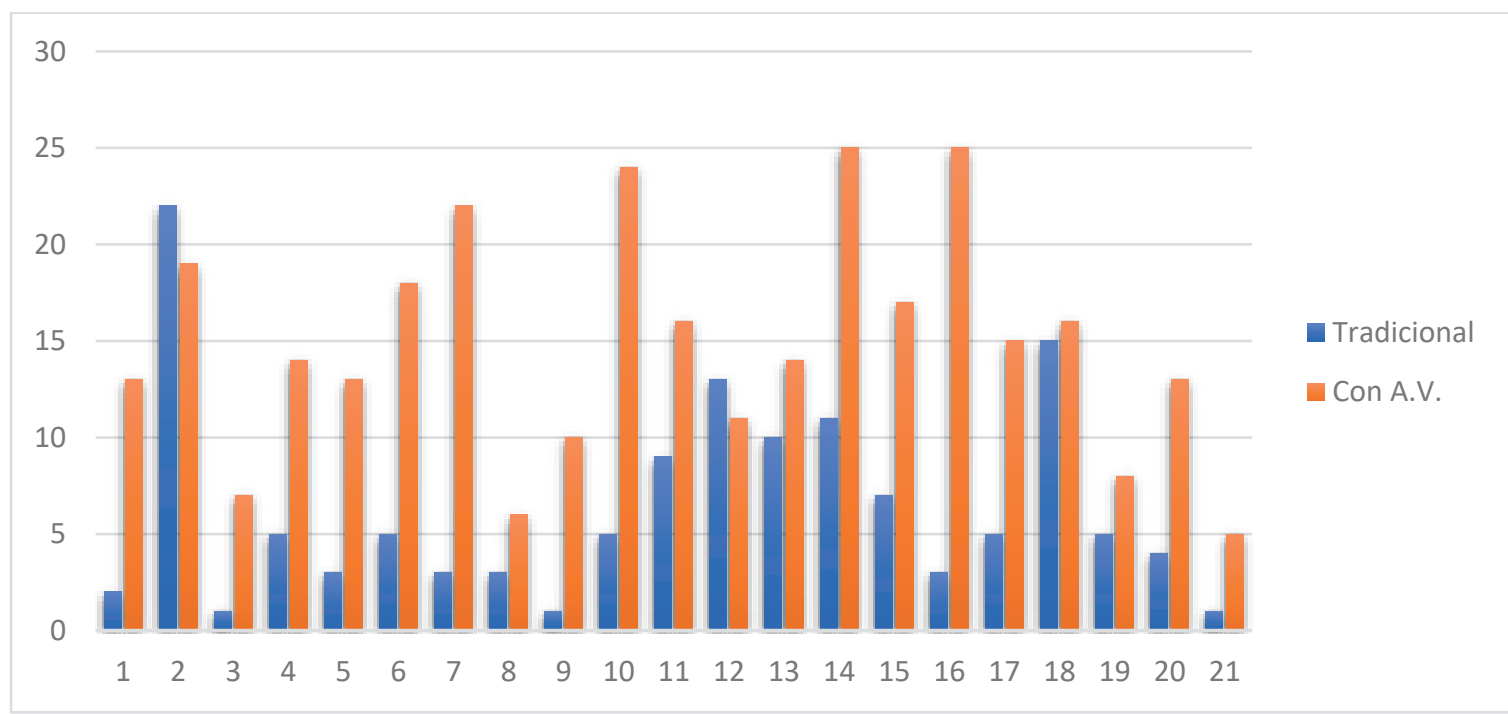

Figura 2. Resultados comparativos del rendimiento de los 30 alumnos de ambos grupos (clase tradicional y clase con AV) para cada una de las 21 preguntas del TUG-K.

Fuente: Elaboración propia.

Tabla 3. Coeficiente de correlación biserial puntual.

\begin{tabular}{|l|c|c|}
\hline & Herramienta & Aciertos $(\%)$ \\
\hline Herramienta & 1 & $0.809^{*}$ \\
\hline Aciertos (\%) & $0.809^{*}$ & 1 \\
\hline
\end{tabular}

* La correlación es significativa en el nivel 0.01 (bilateral).

Fuente: Elaboración propia.

Tabla 4. Comparaciones estadísticas del porcentaje de aciertos de los objetivos y del TUG-K.

\begin{tabular}{|c|c|c|c|c|c|c|}
\hline \multirow{2}{*}{\multicolumn{2}{|c|}{ Comparación }} & \multicolumn{2}{|c|}{ Aciertos (\%) } & \multirow{2}{*}{ Sig. * } & \multirow{2}{*}{$\mathbf{t}$} & \multirow{2}{*}{ Sig. (lateral derecha) $)^{* *}$} \\
\hline & & Con AV & Sin AV & & & \\
\hline \multirow{7}{*}{ Objetivos } & 1 & $46.7 \%$ & $20.0 \%$ & 0.106 & 3.911 & $<0.0001$ \\
\hline & 2 & $65.7 \%$ & $33.3 \%$ & 0.419 & 4.822 & $<0.0001$ \\
\hline & 3 & $47.7 \%$ & $26.7 \%$ & 0.008 & 2.877 & 0.006 \\
\hline & 4 & $69.0 \%$ & $11.0 \%$ & 0.033 & 9.252 & $<0.0001$ \\
\hline & 5 & $64.3 \%$ & $30.0 \%$ & 0.569 & 4.466 & $<0.0001$ \\
\hline & 6 & $20.0 \%$ & $5.7 \%$ & $<0.0001$ & 2.644 & 0.012 \\
\hline & 7 & $32.3 \%$ & $21.0 \%$ & 0.767 & 1.86 & 0.068 \\
\hline \multicolumn{2}{|c|}{ TUG-K } & $49.4 \%$ & $21.1 \%$ & 0.027 & 10.49 & $<0.0001$ \\
\hline
\end{tabular}

* Prueba de Levene de homogeneidad de varianzas.

${ }^{* *}$ Prueba de t de Student lateral derecha con la que se intenta probar si el porcentaje de aciertos es superior con la implementación de AV en comparación a la clase tradicional.

Fuente: Elaboración propia. 
puede ser un reflejo del nivel de educación de los estudiantes del país, ya que, según Larenas (30 de abril de 2019), citando un informe de la Organización para la Cooperación y el Desarrollo Económico, el Perú ocupa el último lugar en rendimiento escolar en Latinoamérica; además, el $68.5 \%$ de los estudiantes peruanos no alcanza el nivel básico $(60 \%)$ en ciencias.

\section{Ganancia de Hake}

Con respecto a la ganancia de Hake global de $32,5 \%$ en las pruebas TUG-K a los alumnos que recibieron la clase tradicional en relación a los alumnos que recibieron en su clase el $\mathrm{AV}$, esta constituye una ganancia similar a la de los estudios de Sandoval et al. (2017) y Navarrete et al. (2015), los cuales tuvieron ganancias de Hake superiores al $40 \%$ y $42.86 \%$, respectivamente. De acuerdo con Hake (1998), una ganancia entre el $30 \%$ y el $70 \%$ es media, lo que significa que implementar el $\mathrm{AV}$ en las clases prácticas de cinemática tiene un impacto medio en el NCGC en los alumnos de ingeniería de la Universidad de Huánuco.

\section{Correlación entre el tipo de clase y el porcentaje de aciertos}

Según la Tabla 3, existe una fuerte correlación (coeficiente de correlación biserial puntual $r_{b p}=0,809$ ) altamente significativa $(p<0,0001)$ entre el tipo de clase impartida (tradicional y con AV) y el porcentaje de preguntas acertadas del TUG-K. Esto debido a la mayor interacción del alumno con el experimento en la clase con AV, en comparación con la clase tradicional, ya que fueron los mismos estudiantes los que se encargaron de la instalación del fondo negro, de la barra de calibración y de la cámara de video; además, ellos hicieron los lanzamientos de la bola de hule.

Aquí vale añadir lo señalado por Castorina (2001), quien indica que las teorías constructivistas sostienen que las personas pueden aprender al interactuar con objetos y otros individuos, en el contexto de una cultura, y que, en esta interacción, los individuos transforman la realidad (no literalmente, sino dándole un significado) al tiempo que modifican los patrones de la misma, ya que dichos patrones son una representación de una situación específica o de un concepto que nos posibilita manipularlos internamente y encarar situaciones idénticas o parecidas a las reales. Entonces, se puede afirmar que la clase práctica que implementa el AV aumenta la participación e interacción de los alumnos, lo que ayuda a que ellos atribuyan sentido a los experimentos realizados, por medio de esquemas aprendidos en sus clases teóricas, para que puedan enfrentar situaciones similares a las de la realidad.

Influencia de la clase de AV en el nivel de preguntas acertadas del TUG-K en comparación con la clase tradicional

Al realizar la prueba de t de Student para dos muestras independientes (clase tradicional y con AV) a un nivel de $\alpha=0.05$, se encontró evidencia estadística altamente significativa ( $p<0,0001)$ para afirmar que el porcentaje de preguntas correctas del TUG-K es mayor cuando se implementa el AV en las clases prácticas ( $49.4 \%$ de aciertos) en comparación con la clase tradicional (21.1\% de aciertos). Estos resultados son coherentes con los estudios de Sandoval et al (2017), Navarrete et al (2015), Adams y Shrum (1990) y Ribotta et al (2009), quienes encontraron que el porcentaje de aciertos del TUG-K es superior cuando se aplica el AV en las clases prácticas. Entonces, se aprecia que el AV mejora significativamente el NCGC de alumnos de ingeniería.

Asimismo, según Beichner (1990), los experimentos de laboratorio en tiempo real, basados en microcomputadoras, permiten que los estudiantes "vean" y, al menos en los ejercicios de cinemática, "sientan" la conexión entre un evento físico y su representación gráfica.

\section{RECOMENDACIONES}

Debido al bajo NCGC y al bajo rendimiento escolar del país, se recomienda aplicar las técnicas del AV descritas en el presente artículo, ya que su costo es bajo y su implementación es relativamente sencilla.

En trabajos posteriores se puede emplear el AV para las clases de mecánica en general, no solo a nivel universitario, sino también a nivel escolar, como se evidencia en el estudio de Guidugli, Fernández y Benegas (2004).

Se recomienda que los docentes innoven sus clases, puesto que una pequeña variación en el desarrollo experimental de la práctica tiene una gran influencia en la comprensión de gráficos en cinemática.

\section{CONCLUSIONES}

1. El NCGC de los alumnos de ingeniería de la Universidad de Huánuco que llevaron el curso de Física I es muy bajo (solo $21.1 \%$ de aciertos del TUG-K). 
2. La implementación del AV en las clases prácticas del curso de Física I de alumnos de ingeniería de la Universidad de Huánuco tuvo un impacto medio, con una ganancia de Hake de $32.5 \%$ en el NCGC (preguntas acertadas del $21.1 \%$ al $49.4 \%)$.

3. Existe una fuerte correlación altamente significativa entre el tipo de clase impartida (tradicional y con AV) y el NCGC, lo cual se refleja en el porcentaje de preguntas acertadas del TUG-K.

4. EI NCGC es mayor cuando se implementó el $\mathrm{AV}$ en las clases prácticas en comparación con las tradicionales.

\section{REFERENCIAS BIBLIOGRÁFICAS}

[1] Adams, D. y Shrum, J. (1990). The effects of microcomputer-based laboratory exercises on the acquisition of line graph construction and interpretation skills by high school biology students. Journal of Research in Science Teaching, 27(8), 777-787.

[2] Beichner, R. (1990). The effect of simultaneous motion presentation and graph generation in a kinematics lab. Journal of Research in Science Teaching, 27(8), 803-815.

[3] Beichner, R. (1994). Testing student interpretation of kinematics graphs. American Journal of Physics, 62(8), 750-762.

[4] Castorina, J. (2001). Los problemas conceptuales del constructivismo y sus relaciones con la educación. En Baquero, R. et al., Debates Constructivistas (pp. 21-46). Buenos Aires, Argentina: Aique.

[5] Cid, X. (2011). Investigations in the impact of visual cognition and spatial ability on student comprehension in physics and space science. (Tesis de doctorado), University of Texas at Arlington, Estados Unidos.

[6] Elorza, H. (1999). Estadística para las ciencias sociales y del comportamiento. México D. F., México: Oxford University Press.

[7] Guidugli, S., Fernández, C. y Benegas, J. (2004). Aprendizaje activo de la cinemática lineal y su representación gráfica en la escuela secundaria. Enseñanza de las ciencias. Revista de investigación y experiencias didácticas, 22(3), 463-472.
[8] Hake, R. (1998). Interactive-engagement versus traditional methods: A six-thousandstudent survey of mechanics test data for introductory physics courses. American Journal of Physics, 66(1), 64-74.

[9] Larenas, O. (30 de abril de 2019). Perú quedó en último puesto en Sudamérica en rendimiento escolar. Agencia de Noticias Señal Alternativa. Recuperado de http://senalalternativa.com/ noticias/peru-quedo-en-ultimo-puesto-ensudamerica-en-rendimiento-escolar.html.

[10] Meneses, J., Niño, J. y García, F. (2017). Kine-uis: modelamiento de video para la adquisición de la velocidad y aceleración angular instantánea de un sólido rígido. Revista Colombiana de Tecnologías de Avanzada, 2(30), 1-9.

[11] Navarrete, L., Almaguer, J., Navarrete, F. y Flores, M. (2015). El análisis de video como alternativa para la integración de teoría y práctica en los cursos introductorios de Física. Latin-American Journal of Physics Education, 9(3), 1-8.

[12] Pontes, A. (2005). Aplicaciones de las tecnologías de la información y de la comunicación en la educación científica. Primera parte: funciones y recursos. Revista Eureka sobre Enseñanza y Divulgación de las Ciencias, 2(1), 2-18.

[13] Ribotta, S., Pesetti, M. y Pereyra, S. (2009). Las tecnologías de la información y comunicación (TICs) aplicadas a la comprensión de gráficos en Cinemática. Formación Universitaria, 2(5), 23-30.

[14] Sandoval, M., Mora, C. y Suárez, C. (2017). Estrategia enseñanza-aprendizaje basada en experimentos (ABE) para mejorar la comprensión de gráficas en Cinemática. Latin-American Journal of Physics Education, 11(3), 1-8. 


\title{
Assessment of the Influence and Impact of Video Analysis on Students' Understanding of Graphs in Kinematics: A Low-Cost and Easy-to-Implement Alternative
}

\author{
CÉSAR Rosas ECHEVARría ${ }^{1}$ \\ Jorge Hilario CÁRDENAS ${ }^{2}$ \\ Roberto Perales Flores ${ }^{3}$ \\ Alberto Cerna Cueva ${ }^{4}$ \\ Justina Isabel Prado Juscamaita 5
}

ReCibido: 09/09/2019 Aceptado: 21/01/2020 Publicado: 16/10/2020

\begin{abstract}
This study demonstrates the benefits of implementing video analysis (VA) in physics instruction. It aims at demonstrating that the implementation of $\mathrm{VA}$, in addition to being a low-cost and easy-to-use technique, significantly improves students' understanding level of graphs in kinematics (ULGK). The study compares the results obtained after applying the Test of Understanding Graphs in Kinematics (TUG-K) on a practical kinematics class in a traditional lecture against one conducted using $\mathrm{VA}$, in order to evaluate the influence of VA on students ULGK. The population was comprised of 60 students, 30 per group, from the School of Engineering of the Universidad de Huánuco. The results show that a strong, highly significant point-biserial correlation $\left(r_{p b}=0.809\right)(p$ $<0.0001)$ exists between these two variables, that VA has an average impact on the ULGK (Hake $=32.5 \%)$ and that the ULGK is significantly higher $(p<0.0001)$ when $V A$ is applied compared to traditional instruction.
\end{abstract}

Keywords: video analysis; understanding level of graphics in kinematics; TUG-K; point-biserial correlation; tracking.

\section{INTRODUCTION}

The ability to work with graphs is a basic skill of the scientist (Beichner, 1994); however, studies show that this is not a skiII most students, who study kinematics in their academic formation, possess. According to Cid (2011), a correlation exists between performance in the fields of science, technology, engineering and mathematics, and the ability to work with graphs. Moreover, according to Pontes (2005), it is known that there is a growing influence of information and communication technologies (ICT) on science education at secondary and university level.

One way to assess students' understanding level of graphs in kinematics (ULGK) is by means of the Test of Understanding Graphs in Kinematics (TUG-K), proposed by Beichner (1994), who applied it to 524 high school and college students, and obtained an average of 8.5 out of 21 questions - only $40 \%$ of correct answers. Similarly, Ribotta, Pesetti and Pereyra (2009) have demonstrated low ULGK with the TUG-K with 62 students studying Physics I, at the School of Engineering and Social Economic Sciences (FICES) of the Universidad Nacional de San Luis (UNSL), Argentina; in this test, $36.83 \%$ of the answers were correct.
$1 \quad$ Industrial engineer from the Universidad Nacional Hermilio Valdizán (Huánuco, Peru). Master in Project Management. Currently working as Professor on a contract basis at said university and the Universidad de Huánuco. (Huánuco, Peru). ORCID: https://orcid.org/0000-0002-3256-3102

E-mail: crosas@unheval.edu.pe

$2 \mathrm{PhD}$ in Business Management from the Universidad Nacional Hermilio Valdizán (Huánuco, Peru). Currently working as professor at said university. (Huánuco, Peru).

ORCID: https://orcid.org/0000-0001-6627-6489

E-mail: jhilario@unheval.edu.pe

3 Master in Education and Planning from the Universidad Nacional Hermilio Valdizán (Huánuco, Peru). Currently working as professor at said university. (Huánuco, Peru). ORCID: https://orcid.org/0000-0002-2502-9593 E-mail: rperales@unheval.edu.pe

4 Environmental engineer from the Universidad Nacional Agraria de la Selva (Tingo María, Peru). Currently working as an independent consultant. (Tingo María, Peru). ORCID: https://orcid.org/0000-0001-7448-558X E-mail: franco.cerna@unas.edu.pe

$5 \mathrm{PhD}$ in Health Science from the Universidad Nacional Hermilio Valdizán (Huánuco, Peru). Currently working as professor at said university. (Huánuco, Peru).

ORCID:https://orcid.org/0000-0002-6558-4233 E-mail: jprado@unheval.edu.pe 
On the other hand, the traditional teaching method does not use technological resources, which causes external representations of theoretical concepts to be limited, due to the constraints of human sensory capacities. That is why there are studies that have shown that experience-based learning (EXL) techniques improve the understanding of kinematic graphs; an example of this is the study of Sandoval, Mora and Suárez (2017). These researchers applied the TUG-K to four groups of students (three with the traditional teaching method and one group with EXL) from Environmental, Industrial or Petroleum Engineering specialties who studied at the Instituto Superior Tecnológico de Comalcalco or the Universidad Politécnica del Golfo de México; the result was Hake gain greater than $40 \%$. This experiment showed that EXL significantly improves the ULGK.

In kinematics, the straightforward way to interact and experiment with the problem is by acquiring real-time data from the position-time of an object, for its further analysis using physical models; however, this data acquisition is only possible if technological tools are used. Video analysis (VA) is one of these tools, where a series of static photographic images, when displayed in sequential order, display a moving image, enabling the construction of position-time graphs (Navarrete, Almaguer, Navarrete \& Flores, 2015). The basics of VA are not new concepts, but this technique has been recently applied in teaching. A few years ago, technological resources were considered too unconventional to be widely applied in teaching and learning processes, due to their complexity and price; now, the use of easily accessible and low cost tools is feasible.

Finally, among the research studies about the teaching process using VA technique for the understanding of graphs in kinematics, is that of Navarrete et al. (2015), who worked with six groups of Industrial Engineering and Chemical Engineering of the Centro Universitario de Ciencias Exactas e Ingenierías from the Universidad de Guadalajara taking laboratory physics. In that research, TUG-K was applied before and after the integration of VA into classes during the February-June 2013 semester, and resulted in an average Hake gain of $42.86 \%$, from which it was concluded that the integration of VA in physics instruction improves the ULGK. Another study in which video analysis is applied is the study by Ribotta et al, (2009), who worked with 62 students of the UNSL FICES Physics I course, who answered the TUG-K at the beginning and end of the class. Students were divided into 3 experimental groups (control group, 21; DataStudio group, 20; and VideoPoint group, 21), obtaining Hake gains of $5.0 \%, 21.8 \%$ and $17.8 \%$ respectively. Thus, it was concluded that the application of ICT contributes to improving pedagogical-didactic aspects in the training of engineers.

\section{MATERIALS AND METHODS}

\section{Population and sample delimitation}

To assess the impact of TUG-K on the interpretation of graphs in kinematics, this research compared traditional instruction against a class that integrated VA. Thirty architecture students and 30 civil engineering students were selected for each class, respectively; both groups were comprised of first semester students enrolled in the Physics I course at the School of Engineering of the Universidad de Huánuco. As the number was initially higher, the sample was selected using a screening process; only those students who regularly attended classes prior to the kinematics topic and those who participated most during the class on that topic were selected.

\section{Implementation of VA technique}

As per the study by Meneses, Niño and García (2017), the procedure and implementation of VA technique consisted on the following:

- Contrast background: VA technique analyzes the pixels of each frame, for this reason, to ease the contrast between the study object (a rubber ball) and the background, a rectangular black piece of fabric of $2 \times 3 \mathrm{~m}$ was stretched out on a wooden frame.

- Determination of coordinate axis: This was done by identifying the bottom left corner of the of the black cloth background. Although the ball tosses were made in the first quadrant of the axis, this served as a reference of motion.

- Calibration bar: A one-meter long wooden bar was used as a calibration bar and was placed on one side of the black fabric. Thus, regardless of the distance at which the video was recorded, it was possible to calculate the displacements of the object along the XY-plane.

- Detecting the presence of a color object (the rubber ball) using computer vision techniques: Placing a black background was helpful as the contrast assisted the discrimination process of color selection with the OpenCV tracking library. Phyton 2.7 programming language was used. 
- Track the ball as it moved in the film frames: A cell phone video recording was used. Information regarding the rubber ball position was specific, albeit this is an object that has a surface; to overcome this, we worked with the center of the figure of the ball, which was calculated from its pixels. The video was tracked for intervals of 100 milliseconds, and data were exported to Excel, where the graphs were created ( $s$ vs. $t),(v$ vs. $t)$ and (a vs. t).

\section{Preparation of study class}

Figure 1 shows a diagram of the study classes taught below.

\section{TUG-K}

TUG-K is intended to be a diagnostic tool for ULGK. This test consists of seven evaluation objectives and three questions per objective, 21 questions in total. Details are shown in Table 1:

\section{Statistical design}

In order to determine if a relationship exists between the received instruction (traditional or $\mathrm{AV}$ ) and the percentage of correct answers on the TUG-K, the point-biserial correlation coefficient was applied. In general, biserial correlation is a measure of association between two continuous variables, which are normally distributed; to facilitate the process, one of them is dichotomized (yes-no, false-true, masculine-feminine, married-single, good-bad, among others). The dichotomous variable is assumed to be discrete or discontinuous when it comes to relating to the one that remains continuous; the most appropriate coefficient for this purpose is that of point-biserial correlation $\left(r_{p b}\right)$ (Elorza, 1999). For this study, instruction received will be the dichotomous variable ( 0 for the traditional instruction and 1 for the instruction with VA). The point-biserial correlation coefficient was calculated as follows:

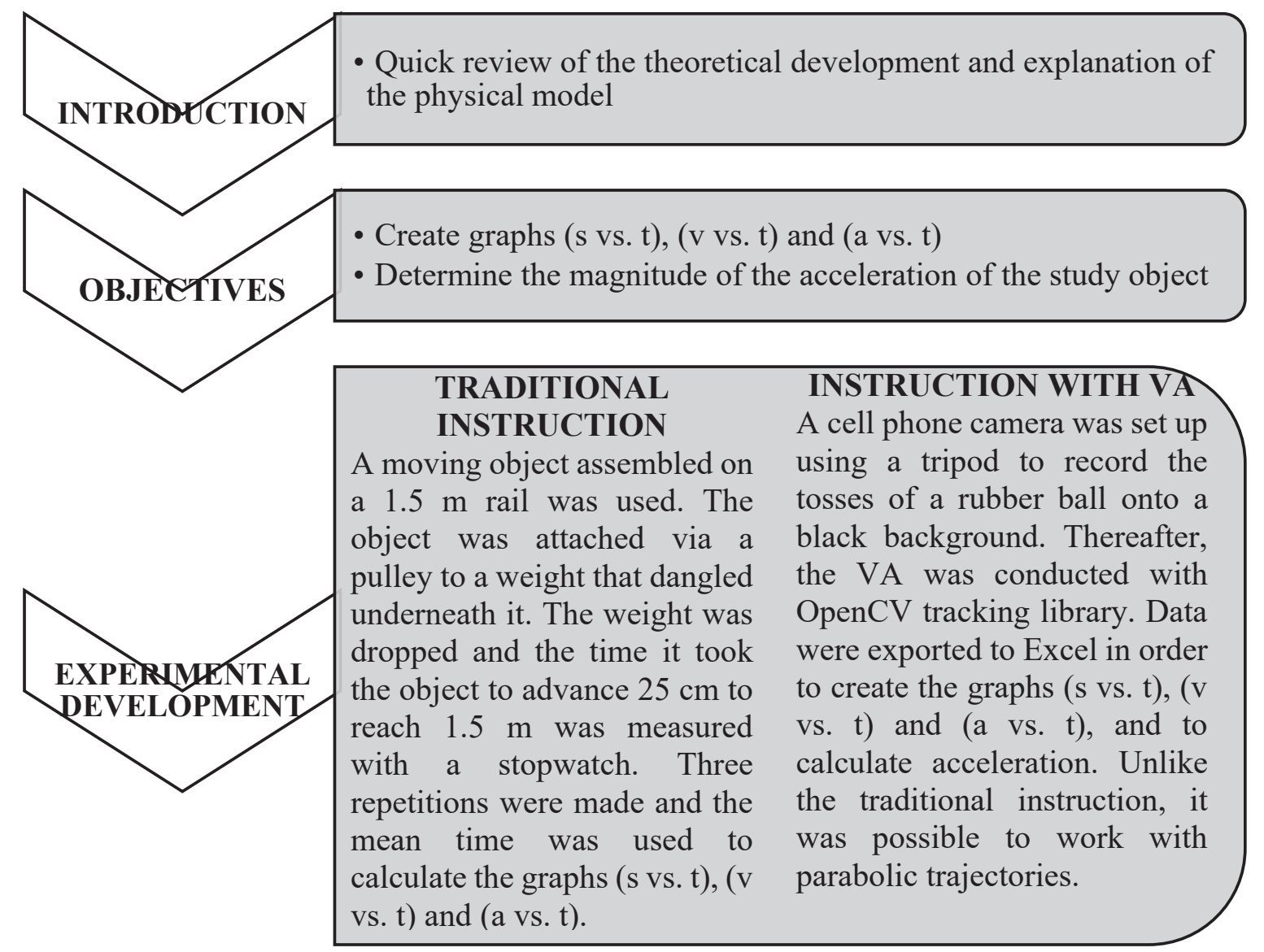

Figure 1. Procedure of the practical kinematics classes: traditional and VA technique. 
Table 1. Objectives of TUG-K.

\begin{tabular}{|c|c|c|}
\hline No. & Given: & The student will: \\
\hline 1 & a position-time graph & determine the speed \\
\hline 2 & a speed-time graph & determine the acceleration \\
\hline 3 & a speed-time graph & determine the displacement \\
\hline 4 & an acceleration-time graph & select another corresponding graph \\
\hline 5 & any kinematics graph & select the textual description \\
\hline 6 & any kinematics graph & select the corresponding graph \\
\hline 7 & a description of motion & \\
\hline
\end{tabular}

Source: Beichner (1994).

$$
r_{b p}=\frac{\bar{X}_{p}-\bar{X}}{S_{x}} \sqrt{\frac{p}{q}}
$$

Where:

$\overline{\mathrm{X}}_{\mathrm{p}}$ is the average number of correct answers from students who received instruction with the VA technique.

$\bar{X}$ is the total average number of correct answers of all the students in both groups.

$S_{x}$ is the variance of the study sample.

$p$ and $q$ are the proportion of students who received instruction using VA and traditional technique respectively. As there are 30 students in each group, then $p=q=0.5$.

A one-tailed Student's $t$ test with a significance value of $\alpha=0.05$ was conducted upon determining correlation in order to determine which of the classes taught (traditional and with VA) obtains a higher percentage of correct answers. Therefore, the same procedure was followed for the TUG-K objectives.

\section{RESULTS}

\section{Correlation between class taught and per- centage of correct answers on the TUG-K}

After the two study groups (traditional and with VA) received instruction, the TUG-K was applied to both over a period of 45 minutes. Figure 2 shows the number of correct answers for each question posed in the aforementioned test.

On the other hand, the percentage of correct answers per TUG-K item of both study groups is shown in Table 2. Traditional instruction obtained $21.1 \%$, whereas instruction with VA technique obtained $49.4 \%$. A mean Hake gain of $32.5 \%$ for both groups was obtained.
Table 2. Percentage of correct answers per TUG-K item for the group with traditional instruction versus the group with VA instruction, as well as normalized Hake gain.

\begin{tabular}{|c|c|c|c|}
\hline Item & Traditional & With VA & Hake gain \\
\hline 1 & $6.7 \%$ & $43.3 \%$ & $39.3 \%$ \\
\hline 2 & $73.3 \%$ & $63.3 \%$ & $-37.5 \%$ \\
\hline 3 & $3.3 \%$ & $23.3 \%$ & $20.7 \%$ \\
\hline 4 & $16.7 \%$ & $46.7 \%$ & $36.0 \%$ \\
\hline 5 & $10.0 \%$ & $43.3 \%$ & $37.0 \%$ \\
\hline 6 & $16.7 \%$ & $60.0 \%$ & $52.0 \%$ \\
\hline 7 & $10.0 \%$ & $73.3 \%$ & $70.4 \%$ \\
\hline 8 & $10.0 \%$ & $20.0 \%$ & $11.1 \%$ \\
\hline 9 & $3.3 \%$ & $33.3 \%$ & $31.0 \%$ \\
\hline 10 & $16.7 \%$ & $80.0 \%$ & $76.0 \%$ \\
\hline 11 & $30.0 \%$ & $53.3 \%$ & $33.3 \%$ \\
\hline 12 & $43.3 \%$ & $36.7 \%$ & $-11.8 \%$ \\
\hline 13 & $33.3 \%$ & $46.7 \%$ & $20.0 \%$ \\
\hline 14 & $36.7 \%$ & $83.3 \%$ & $73.7 \%$ \\
\hline 15 & $23.3 \%$ & $56.7 \%$ & $43.5 \%$ \\
\hline 16 & $10.0 \%$ & $83.3 \%$ & $81.5 \%$ \\
\hline 17 & $16.7 \%$ & $50.0 \%$ & $40.0 \%$ \\
\hline 18 & $50.0 \%$ & $53.3 \%$ & $6.7 \%$ \\
\hline 19 & $16.7 \%$ & $26.7 \%$ & $12.0 \%$ \\
\hline 20 & $13.3 \%$ & $43.3 \%$ & $34.6 \%$ \\
\hline 21 & $3.3 \%$ & $16.7 \%$ & $13.8 \%$ \\
\hline
\end{tabular}

Source: Prepared by the authors.

According to Table 3, the point-biserial correlation coefficient is 0.809 , a value close to one; thus, it can be said a strong positive and highly significant correlation exists between the type of instruction provided and the percentage of correct answers on the TUG-K. 


\section{Comparison of the percentage of correct} answers on the TUG-K in both study groups

Table 4 below shows the percentage of correct answers per TUG-K objective.

\section{DISCUSSION}

\section{Diagnostic}

The percentage of TUG-K questions correctly answered by the group that did not receive instruction with VA was $21.1 \%$, a value lower than those in the studies by Beichner (1994) and Ribotta et al. (2009), which obtained percentages of $40 \%$ and $36.83 \%$, respectively. This might be a reflection of the level of education of the country, as, according to Larenas (April 30, 2019), citing a report by the Organization for Economic Co-operation and Development, Peru ranks last in Latin America in terms of school performance. In addition, $68.5 \%$ of Peruvian students do not reach the basic level $(60 \%)$ in science.

\section{Hake gain}

The overall Hake gain score of $32.5 \%$ on the TUG-K of the traditional class group in relation to the VA class group constitutes a gain similar to that of Sandoval et al. (2017) and Navarrete et al. (2015), which obtained Hake gains higher than $40 \%$ and $42.86 \%$ respectively. According to Hake (1998), a gain between $30 \%$ and $70 \%$ is average, which means that implementing VA in practical kinematics classes has an average impact on the ULGK of engineering students at the Universidad of Huánuco.

\section{Correlation between type of class and correct answers percentage}

According to Table 3, there is a strong correlation (point-biserial correlation coefficient $r_{p b}=0.809$ ) highly significant $(p<0.0001)$ between the type of instruction (traditional and with VA) and the percentage of TUG-K correct answers. This is due to the greater interaction of students with the experiment in the VA class, compared to the traditional class, considering

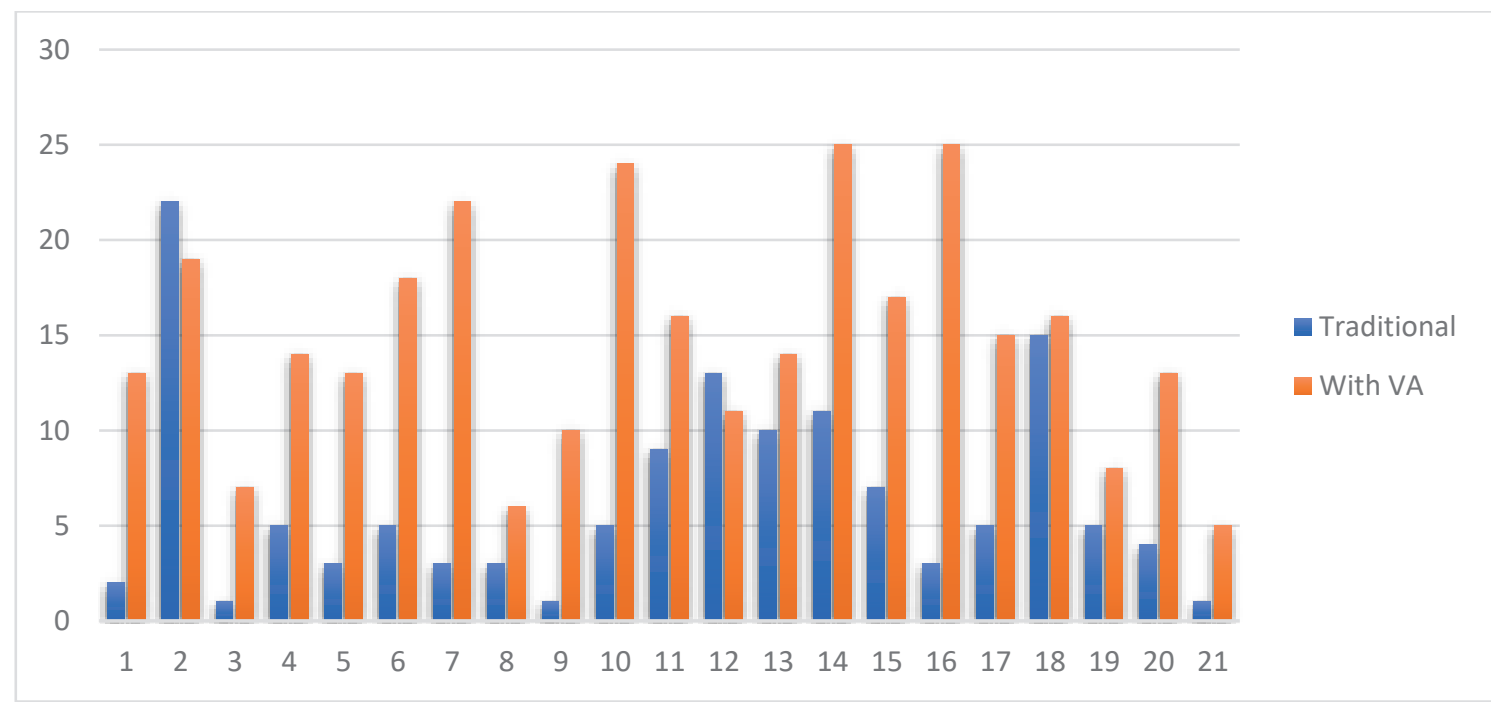

Figure 2. Comparative results of the performance of the 30 students from both groups (traditional and with VA) for each of the 21 TUG-K questions.

Source: Prepared by the authors.

Table 3. Point-biserial correlation coefficient.

\begin{tabular}{|l|c|c|}
\hline & Tool & Correct answers (\%) \\
\hline Tool & 1 & $0.809^{*}$ \\
\hline Correct answers (\%) & $0.809^{*}$ & 1 \\
\hline
\end{tabular}

* Correlation is significant at the 0.01 level (2-tailed).

Source: Prepared by the authors. 
Table 4. Statistical comparison of correct answers regarding the objectives and TUG-K.

\begin{tabular}{|c|c|c|c|c|c|c|}
\hline \multirow{2}{*}{\multicolumn{2}{|c|}{ Contrast }} & \multicolumn{2}{|c|}{ Correct answers (\%) } & \multirow{3}{*}{$\frac{\text { Sig. }}{0.106}$} & \multirow{3}{*}{$\begin{array}{c}\mathbf{t} \\
3.911\end{array}$} & \multirow{3}{*}{$\begin{array}{c}\text { Sig. (right tail) })^{\star *} \\
<0.0001\end{array}$} \\
\hline & & \multirow{2}{*}{$\begin{array}{c}\text { With VA } \\
46.7 \%\end{array}$} & \multirow{2}{*}{$\begin{array}{c}\text { Without VA } \\
20.0 \%\end{array}$} & & & \\
\hline \multirow{7}{*}{ Objectives } & 1 & & & & & \\
\hline & 2 & $65.7 \%$ & $33.3 \%$ & 0.419 & 4.822 & $<0.0001$ \\
\hline & 3 & $47.7 \%$ & $26.7 \%$ & 0.008 & 2.877 & 0.006 \\
\hline & 4 & $69.0 \%$ & $11.0 \%$ & 0.033 & 9.252 & $<0.0001$ \\
\hline & 5 & $64.3 \%$ & $30.0 \%$ & 0.569 & 4.466 & $<0.0001$ \\
\hline & 6 & $20.0 \%$ & $5.7 \%$ & $<0.0001$ & 2.644 & 0.012 \\
\hline & 7 & $32.3 \%$ & $21.0 \%$ & 0.767 & 1.86 & 0.068 \\
\hline \multicolumn{2}{|c|}{ TUG-K } & $49.4 \%$ & $21.1 \%$ & 0.027 & 10.49 & $<0.0001$ \\
\hline
\end{tabular}

* Levene's test for homogeneity of variance.

${ }^{* *}$ Right-tailed Student's t test that attempts to test if the percentage of correct answers is higher with the implementation of VA compared to traditional instruction.

Source: Prepared by the authors.

that the students themselves were in charge of the installation of the black background, the calibration bar and the video camera. Furthermore, they were responsible for tossing the rubber ball.

It is worth adding the statement made by Castorina (2001), who indicates that constructivist theories suggest that people can learn by interacting with objects and other individuals, in the context of a culture, and that, in this interaction, individuals transform reality (not literally, but giving it a meaning) while modifying its patterns. These patterns are representations of specific situations or concepts that enable us to manipulate them internally and to deal with situations identical or similar to reality. Thus, it can be said that practical instruction that implements VA increases the participation and interaction of students, which helps them to attribute meaning to the experiments performed, by means of learned schemes in their theoretical classes, so that they can face situations similar to reality.

\section{Influence of VA instruction on the level of co- rrect TUG-K answers compared to traditional instruction}

Highly significant statistical evidence $(p<0.0001)$ was found upon performing the Student's $t$ test for two independent samples (traditional instruction and with VA) at a significance level of $\alpha=0.05$; thus, it can be stated that the percentage of TUG-K correct answers is higher when VA is implemented in practical classes ( $49.4 \%$ of correct answers) compared to traditional instruction $(21.1 \%$ of correct answers). These results are consistent with the studies of Sandoval et al. (2017), Navarrete et al. (2015), Adams and Shrum (1990) and Ribotta et al. (2009), who found that the percentage of TUG-K correct answers is higher when VA is applied in practical classes. Thus, the VA significantly improves the ULGK of engineering students.

Likewise, according to Beichner (1990), real-time microcomputer-based laboratory allow students to "see" and, at least in kinematics exercises, "feel" the connection between a physical event and its graphical representation.

\section{RECOMMENDATIONS}

The application of VA techniques described in this article is recommended to reduce the country's low ULGK and school performance, as its implementation is low-cost and relatively simple.

In future works, VA can be used for physics courses in general, not only at university level, but also at the secondary level, as demonstrated by Guidugli, Fernández and Benegas (2004).

It is recommended that professors innovate their instruction methods, as a small variation in the experimental development of practice has a major influence on the understanding of graphs in kinematics.

\section{CONCLUSIONS}

1. The ULGK of engineering students of the Universidad de Huánuco enrolled in Physics I course is very low (only $21.1 \%$ of correct answers on the TUG-K).

2. VA implementation in practical classes of the Physics I course for engineering students of the Universidad de Huánuco had an average impact, with a Hake gain of $32.5 \%$ in ULGK ( $21.1 \%$ to $49.4 \%$ correct answers). 
3. There is a strong highly significant correlation between the instruction methodology (traditional and with VA) and the ULGK, which is reflected in the percentage of correct answers on the TUG-K.

4. The NCGC is higher when VA was implemented in the practical classes compared to those with traditional instruction.

\section{REFERENCES}

[1] Adams, D. \& Shrum, J. (1990). The effects of microcomputer-based laboratory exercises on the acquisition of line graph construction and interpretation skills by high school biology students. Journal of Research in Science Teaching, 27(8), 777-787.

[2] Beichner, R. (1990). The effect of simultaneous motion presentation and graph generation in a kinematics lab. Journal of Research in Science Teaching, 27(8), 803-815.

[3] Beichner, R. (1994). Testing student interpretation of kinematics graphs. American Journal of Physics, 62(8), 750-762.

[4] Castorina, J. (2001). Los problemas conceptuales del constructivismo y sus relaciones con la educación. En Baquero, $\mathrm{R}$. et al., Debates Constructivistas (pp. 21-46). Buenos Aires, Argentina: Aique.

[5] Cid, X. (2011). Investigations in the impact of visual cognition and spatial ability on student comprehension in physics and space science. (Doctoral thesis), University of Texas at Arlington, United States.

[6] Elorza, H. (1999). Estadística para las ciencias sociales y del comportamiento. México D. F., Mexico: Oxford University Press.

[7] Guidugli, S., Fernández, C. \& Benegas, J. (2004). Aprendizaje activo de la cinemática lineal y su representación gráfica en la escuela secundaria. Enseñanza de las ciencias. Revista de investigación y experiencias didácticas, 22(3), 463-472.
[8] Hake, R. (1998). Interactive-engagement versus traditional methods: A six-thousandstudent survey of mechanics test data for introductory physics courses. American Journal of Physics, 66(1), 64-74.

[9] Larenas, O. (April 30, 2019). Perú quedó en último puesto en Sudamérica en rendimiento escolar. Agencia de Noticias Señal Alternativa. Retrieved from http://senalalternativa.com/ noticias/peru-quedo-en-ultimo-puesto-ensudamerica-en-rendimiento-escolar.html.

[10] Meneses, J., Niño, J. \& García, F. (2017). Kine-uis: modelamiento de video para la adquisición de la velocidad y aceleración angular instantánea de un sólido rígido. Revista Colombiana de Tecnologías de Avanzada, 2(30), 1-9.

[11] Navarrete, L., Almaguer, J., Navarrete, F. \& Flores, M. (2015). El análisis de video como alternativa para la integración de teoría y práctica en los cursos introductorios de Física. Latin-American Journal of Physics Education, 9(3), 1-8.

[12] Pontes, A. (2005). Aplicaciones de las tecnologías de la información y de la comunicación en la educación científica. Primera parte: funciones y recursos. Revista Eureka sobre Enseñanza y Divulgación de las Ciencias, 2(1), 2-18.

[13] Ribotta, S., Pesetti, M. \& Pereyra, S. (2009). Las tecnologías de la información y comunicación (TICs) aplicadas a la comprensión de gráficos en Cinemática. Formación Universitaria, 2(5), 23-30.

[14] Sandoval, M., Mora, C. \& Suárez, C. (2017). Estrategia enseñanza-aprendizaje basada en experimentos (ABE) para mejorar la comprensión de gráficas en Cinemática. LatinAmerican Journal of Physics Education, 11(3), 1-8. 